Revisão

Revisão

\title{
Força e potência muscular para autonomia funcional de idosos: uma breve revisão narrativa
}

\section{Strength and Muscular Power for Functional Autonomy of the Elderly: a Brief Narrative Review Study}

\begin{abstract}
Mauro Lúcio Mazini Filho ${ }^{\S 1} \mathrm{MS}$; Gabriela Rezende de Oliveira Venturini ${ }^{2} \mathrm{MS}$; Juliana Brandão Pinto de Castro $^{2}$ MS; Alessandra Rodrigues da Silveira ${ }^{3}$; Rodrigo Marçal de Souza ${ }^{3}$; José Mantovani Neto ${ }^{4}$ MD; Maria Elisa Caputo Ferreira ${ }^{1} \mathrm{PhD}$
\end{abstract}

Recebido em: 01 de julho de 2018. Aceito em: 04 de setembro de 2018.

Publicado online em: 27 de setembro de 2018.

\section{Resumo}

Introdução: 0 envelhecimento populacional é um processo em andamento e o tema vem despertando a atenção de autoridades e profissionais de diversas áreas, uma vez que tal fenômeno acarreta perda progressiva e generalizada da força e da potência muscular, entre outros efeitos deletérios. Nesse contexto, cresce o número de trabalhos científicos destacando a importância do treinamento de força e de potência muscular na autonomia funcional do idoso.

Objetivo: Levantar na literatura os efeitos dos treinamentos de força e potência muscular na autonomia funcional de indivíduos idosos.

Métodos: Conduziu-se uma pesquisa de artigos na íntegra nas bases de dados LILACS, Biblioteca Virtual de Saúde (BVS), MedLine e PubMed, tendo como descritores: "muscle strength", "muscle power", "functional autonomy" e "aging".

Resultados: A literatura mostra que os treinamentos de força e de potência muscular, desde que tomados os cuidados com a execução e que sejam devidamente quantificados, tornam-se imprescindíveis para a manutenção/melhora da prática das atividades da vida diária contribuindo para a autonomia funcional de idosos.

Conclusão: Os tipos de treinamento analisados podem contribuir para retardar os efeitos deletérios do envelhecimento, interferindo positivamente na qualidade de vida e na autonomia funcional de idosos.

Palavras-chave: força muscular, potência muscular, autonomia funcional, idoso, treinamento de resistência.
Pontos-Chave Destaque
- O treinamento de força na população idosa pode otimizar o desempenho nas atividades da vida diária e melhorar a autonomia funcional. - O treinamento periodizado, que englobe todos os tipos de treinamento e que considere os objetivos, o nível de treinabilidade e os princípios do treinamento desportivo, é fundamental para resultados seguros e eficientes. - A força, a potência muscular e a hipertrofia podem ser desenvolvidas através do treinamento de força.

\footnotetext{
$\S$ Autor correspondente: Mauro Lúcio Mazini Filho - e-mail: personalmau@hotmail.com Afiliações: ${ }^{1}$ Programa de Pós-Graduação em Educação Física, Universidade Federal de Juiz de Fora (UFJF), Brasil; ${ }^{2}$ Programa de PósGraduação em Ciências do Exercício e do Esporte (PPGCEE), Universidade do Estado do Rio de Janeiro (UERJ), Brasil; ${ }^{3}$ Faculdade de Educação Física e Desportos, Universidade Federal de Juiz de Fora (UFJF), Brasil; ${ }^{\circ}$ Médico geriatra, Cataguases (MG), Brasil.
} 


\begin{abstract}
Introduction: Population aging is an ongoing process and it is an issue of interest for authorities and professionals from different areas, since this phenomenon leads to a progressive and generalized loss of strength and muscular power, among other deleterious effects. In this context, the number of scientific studies increases, emphasizing the importance of strength training and muscle power in the functional autonomy of the elderly.

Objective: To gather in the literature the effects of strength training and muscular power on the functional autonomy of elderly individuals.

Methods: We conducted a survey of articles in full in the databases LILACS, Virtual Health Library (VHL), MedLine and PubMed, having as descriptors: "muscle strength", "muscle power", "functional autonomy" and "aging".

Results: The literature shows that strength training and muscular power training, once taken care of with execution and duly quantified, are essential for maintaining / improving the practice of activities of daily living contributing to the functional autonomy of the elderly.

Conclusion: The types of training analyzed can contribute to delay the deleterious effects of aging, positively interfering in the quality of life and the functional autonomy of the elderly.
\end{abstract}

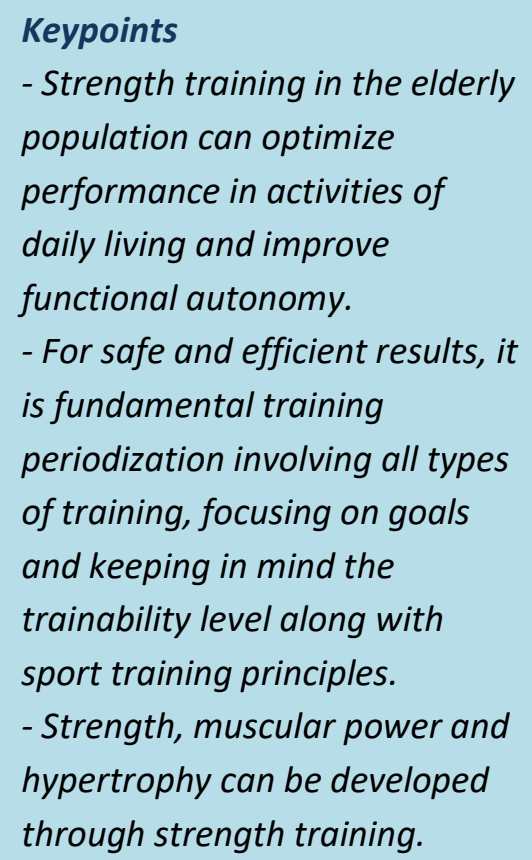

\section{Força e potência muscular para autonomia funcional de idosos: uma breve revisão narrativa}

\section{Introdução}

A população brasileira é de aproximadamente 206 milhões de habitantes. Destes, $12 \%$ são pessoas com idade igual ou superior a 60 anos, as quais são consideradas idosas. Estimativas preveem que, em 2060, este extrato corresponderá a $33 \%$ da população(1).

O envelhecimento tem sido descrito como um processo inerente aos seres vivos e que se expressa pela perda da capacidade de adaptação ao ambiente e diminuição da funcionalidade(2). Nesse processo, ocorre deterioração funcional e estrutural de sistemas fisiológicos, mesmo na ausência de doenças. Essas alterações causam redução da capacidade funcional e mudanças na composição corporal dos idosos(3). O declínio sofrido nas funções neuromusculares e morfológicas resulta em dinapenia (diminuição de força e potência muscular relacionada à idade) e sarcopenia (redução da massa e da função muscular associada à idade)(4-6).

A inatividade física nos idosos pode gerar maior fragilidade muscular e reduzir a motivação, a autoestima(7), a autonomia funcional (AF) e a qualidade de vida(8). Desse modo, a força e potência muscular são parâmetros para aferir a mudança muscular relacionada à idade. A diminuição dessas valências físicas gera implicações na $\mathrm{AF}$ e, consequentemente, na execução de atividades da vida diária - como sentar e levantar de uma cadeira, subir escada, caminhar e vestir$\operatorname{se}(5,9)$.

O treinamento resistido periodizado, respeitando a individualidade biológica e praticado de forma segura, ameniza os efeitos da inatividade física, como a perda de força e de potência muscular(10-12). A força muscular sofre redução de aproximadamente $30 \%$ a $40 \%$ com o envelhecimento. Já o declínio da potência muscular repercute na 
funcionalidade, pois, para realizar as atividades da vida diária, além de realizar o trabalho em força, é necessário que esta seja produzida rapidamente. A queda anual de potência chega à ordem de $3,5 \%$ após os 60 $\operatorname{anos}(13,14)$.

A potência muscular é qualidade física força associada à velocidade. Assim sendo, ela pode ser explicada pela capacidade de produzir a maior quantidade de força na menor fração de tempo possível $(13,15)$. As fibras musculares de contração rápida (tipo II) contribuem no tempo de reação e resposta a situações de emergência. Porém, estas fibras são as mais comprometidas com o envelhecimento(16), podendo levar o idoso à dependência funcional(17).

No treinamento de força, os músculos são movidos ou tendem a se mover contra uma força externa, como pesos livres (barras, halteres) ou máquinas desenvolvidas para exercer resistência. Esse treinamento pode melhorar o desempenho motor (melhor capacidade para correr, arremessar um objeto e saltar) e o desempenho nas AVD (levantar da cama/cadeira, subir escadas, carregar sacolas)(18).

O objetivo do estudo foi reunir na literatura os efeitos do treinamento de força e/ou potência muscular sobre a AF e as implicações para a população idosa.

\section{Métodos}

Trata-se um breve relato sobre o treinamento de força e de potência muscular na AF associada ao envelhecimento e as possíveis implicações, numa perspectiva atual. Conduziu-se uma pesquisa de artigos na íntegra nas bases de dados LILACS, Biblioteca Virtual de Saúde (BVS), MedLine e PubMed, tendo como descritores: "muscle strength", "muscle power", "functional autonomy" $\mathrm{e}$ "aging".

\section{Resultados e Discussão}

Os estudos que compuseram o presente estudo de revisão pertenciam a diferentes áreas da saúde, incluindo Educação Física, Fisioterapia, Medicina(19-26), o que evidencia o caráter multidisciplinar da temática em questão. Observou-se variabilidade no volume e intensidade dos treinamentos prescritos, além de diversidade nos testes de AF aplicados. Apesar dessas diferenças, houve semelhanças, como os efeitos da inatividade física no processo de envelhecimento, conforme se expõe a seguir.

Um estudo com 227 mulheres não sedentárias avaliou força explosiva e potência muscular inercial. Estas valências físicas mostraram-se significativamente menores em mulheres a partir de 50 anos, quando comparadas com estas qualidades físicas aos 18 anos de idade(26). Em força explosiva, as participantes com idade de 18 anos apresentaram média de $26,10 \mathrm{~cm}$ e as da faixa etária de 50 a 79 anos apresentaram média de $16,03 \mathrm{~cm}$. Em potência muscular inercial, as médias obtidas pelas participantes de 18 anos e acima de 50 anos de idade foram de, respectivamente, $36,54 \mathrm{~cm}$ e $28,98 \mathrm{~cm}$. Os menores valores encontrados para força e potência nas participantes com mais idade são explicados por fatores neurais, resultando em alterações na velocidade de contração muscular e na coordenação intra e intermuscular. Outro fator que pode ter acarretado valores menores é a sarcopenia(26), que não foi investigado no estudo.

Dentro de um contex to voltado para a AF do idoso, o treinamento de potência muscular no qual sejam realizados saltos sucessivos não oferece vantagem na relação custo/benefício, tendo em vista as dificuldades da alta demanda motora. É provável que um treinamento resistido, com estratégias mais seguras, ofereça maior grau de especificidade e de velocidade na fase concêntrica e, por conseguinte, seja mais eficiente para o aumento da potência muscular e, consequentemente, contribua para a $\operatorname{AF}$ de idosos(27,29).

Para Moraes et al.(21), os efeitos deletérios do envelhecimento podem ser revertidos com $\mathrm{o}$ treinamento. Os autores realizaram um estudo com 45 idosas, divididas em um grupo controle e três grupos experimentais, com a duração de seis semanas. Comparando os três grupos experimentais nos seguintes tipos de treinamento: potência, força e força máxima. $\mathrm{O}$ grupo que treinou potência teve melhores resultados nos testes de uma repetição em carga máxima (1RM), enquanto o grupo de força reativa teve melhores resultados nos testes de tempo de reação. Isso demonstra que 
as idosas são capazes de melhorar a capacidade de desenvolver força, revelando o treinamento como um elemento capaz de retardar o declínio desta valência com o envelhecimento. Os autores concluíram que a combinação dos três programas de treinamento contribuiu para QV das idosas(21), ressaltando a importância de haver uma periodização adequada, atentando para as especificidades desta população.

Com o intuito de avaliar a força e a potência muscular na AF do idoso, Cabral et al.(25) aplicaram o protocolo do Grupo de Desenvolvimento Latino-americano para Maturidade (GDLAM)(31,32) em 13 idosas. Este protocolo permite verificar o grau de independência funcional em que se encontra o idoso e engloba os testes: caminhada de 10 metros; levantar-se da posição sentada; levantar-se da posição decúbito ventral o mais rápido o possível; sentar e levantar da cadeira e locomover-se pela casa; vestir e tirar uma camisa. Após um período de treinamento resistido com intensidade progressiva de três meses, houve melhora nos valores obtidos nos testes, com diminuição de $14,3 \%$ no tempo. Desse modo, três meses de intervenção, com aumento progressivo da intensidade, promoveram efeitos positivos na AF de idosas, favorecendo a manutenção de um estilo de vida ativo, com menor probabilidade de depender de terceiros para realizar atividades da vida diária.

Ueno et al.(20) encontraram aumento da força muscular em idosos treinados. Os participantes eram orientados a realizar, com o membro superior dominante, o maior número de flexões de cotovelo possíveis dentro de 30 segundos, segurando um halter de 1,84 kg para as mulheres e de 3,63 kg para homens. Após 16 semanas, ocorreu aumento nos níveis de força muscular, porém, não houve melhora nos níveis de AF. Para os autores, ficou claro que o treinamento de força ajudou na manutenção e não na melhora da $\mathrm{AF}$ dos indivíduos e que tal manutenção teve impacto positivo na qualidade de vida dos idosos.

Nesta mesma linha, Mezzaroba e Prati(23), em estudo experimental, avaliaram ao longo de 12 semanas a força muscular de idosas não praticantes de exercícios físicos. Cada sessão de treinamento incluiu exercícios para músculos principais, em três séries com dez repetições e intervalo de 30 segundos, em que a carga foi aumentada em $10 \%$, se notada extrema facilidade de execução. Para avaliar a capacidade funcional, os autores utilizaram um teste específico para este grupo, a fim de testar o desempenho da auto percepção das atividades de vida, respondendo a um questionário com a pontuação classificadas como "muito ruim", "ruim", "médio", "boa" e "muito boa" de acordo com as atividades realizadas com maior independência. Como resultado, obteve-se aumento da massa muscular, do gasto energético e da resistência, diminuição da massa gorda, além da maior disponibilidade dos idosos para as atividades da vida diária em resposta a alterações causada pelo treinamento de força.

Tiggeman et al.(19) submeteram 30 idosas a 12 semanas de treinamento, duas vezes por semana. As idosas foram divididas em dois grupos com o mesmo número de participantes $(n=15)$. Um grupo realizou treinamento de força tradicional e o outro realizou treinamento de potência muscular. Durante as duas primeiras semanas, ambos os grupos realizaram as fases concêntrica e excêntrica dos exercícios em 2 segundos para cada repetição. Após esse período, o grupo potência muscular realizou os movimentos da fase concêntrica o mais rápido possível, mantendo dois segundos para a fase excêntrica. A intensidade dos exercícios foi determinada pela utilização da percepção subjetiva do esforço da escala de Borg. Para avaliar a AF, foi utilizado o teste de caminhada de 6 minutos, o teste de subida de escada, levantar e andar (Timed Up Go: TUG), e o teste de levantar e sentar em uma cadeira. Embora não tenham sido observadas diferenças significativas comparando os grupos nos testes, ocorreram aumentos no desempenho físico após o treinamento em ambos os grupos durante o teste de caminhada de 6 minutos, subida de escada, levantar e andar e sentar em uma cadeira. As principais conclusões do estudo foram que ambos os programas propiciaram aumento de força, potência muscular e desempenho funcional de forma significativa. Esses achados apoiam a eficácia dos treinamentos de força tradicional e de potência muscular, utilizando percepção subjetiva de esforço no controle da carga de 
treinamento, para tornar mais brandos os efeitos deletérios do envelhecimento em idosas.

Segundo Lustosa et al.(22), para manter a independência funcional, entre outros fatores, é necessário um bom desempenho muscular, em velocidades que sejam compatíveis com as diversas atividades da vida diária. Nesse estudo, foi proposto um programa de exercícios para 32 idosas, que foram submetidas a um período de treinamento de dez semanas, com exercícios realizados três vezes por semana durante uma hora. Os exercícios foram voltados para grandes grupamentos musculares dos membros inferiores e com utilização de cargas adequadas por meio do percentual de resistência máxima. Para as cargas, utilizaramse caneleiras de 0,5 a 3,0 kg. Como resultado, ocorreu melhora da potência muscular e diminuição do tempo de realização de tarefas funcionais, confirmado pelos testes realizados: teste de levantar e andar e teste de caminhada de 10 metros, sendo utilizada uma velocidade confortável para verificar as limitações funcionais no dia a dia, como a capacidade de atravessar uma rua. O grupo experimental apresentou melhora no desempenho funcional após o treinamento quando comparado com o grupo controle. A relevância de tais resultados destaca-se, pois, esses testes são preditores de quedas e dependência funcional de idosos(22).

Prado et al.(24) utilizaram o teste de levantar e andar para avaliar o desempenho funcional após uma intervenção de 5 semanas e obtiveram resultados semelhantes ao estudo de Lustosa et al.(22), com uma amostra de quatro idosas entre 60 e 80 anos. Elas conseguiram reduzir e/ou manter (apenas uma idosa manteve os valores iniciais) o tempo de execução do teste. Esses resultados mostraram que melhoras na potência e força muscular se apresentaram relacionadas a melhoras na mobilidade funcional demonstrando que $o$ programa de exercícios resistidos foi eficaz para o aumento do desempenho físico, que faz com que as idosas realizem melhor as atividades da vida diária. Tais resultados alinham-se a estudos presentes na literatura, em que foram encontrados pequenos efeitos favoráveis dos treinamentos de potência e de força sobre resultados funcionais(31). Nesse contexto, foram observados maiores ganhos de força e hipertrofia muscular em idosos como resultado de programas de treinamento de alta intensidade $(\sim 80 \%$ de $1 \mathrm{RM})$ em relação ao treinamento de intensidade leve a moderada $(\sim 45 \% 1 R M)(32)$.

Após análise dos treinamentos de força e potência muscular, percebe-se que ambos promovem benefícios à população idosa, tais como a prevenção da sarcopenia, de quedas e fraturas; melhora do equilíbrio e da marcha; aumento do bem-estar; e melhora na AF. Assim, recomenda-se uma periodização devidamente planejada levando em consideração os objetivos, fases de treinamento, limitações, preferências, necessidades e individualidade dos idosos. Também em relação à periodização, há necessidade de se contemplar as fases de adaptação neural, bem como realizar aumentos progressivos de carga de treino e que, dentro destes, incluir períodos de força e potência muscular de forma que possa atender a melhoria da AF dos idosos.

\section{Pontos fortes e limitações do estudo}

Um ponto forte do estudo foi trazer um conjunto de estudos recentes sobre os métodos de treinamento contra resistência aplicados a idosos para desenvolver qualidades físicas que contribuam com a AF dessa população e, consequentemente, melhorar sua qualidade de vida.

Uma limitação do estudo foi a quantidade reduzida de estudos apresentados, todavia, este fato não prejudicou as análises realizadas, pois, todos os estudos que compuseram a presente revisão trataram-se de estudos experimentais, contribuindo para conhecimento na área da qualidade de vida e saúde de idosos, cuja população aumenta a cada dia.

\section{Conclusão}

Percebemos que várias atividades da vida diária necessitam de velocidade e outras de maiores níveis de força, por isso a recomendação de um protocolo periodizado que englobe a capacidade física força em suas diversas formas de manifestação a fim de desenvolver um treinamento seguro e eficaz. Observa-se, na literatura, que o treinamento de potência tende a ser promissor, desde que 
devidamente planejado para evitar lesões e otimizar os ganhos que se fazem pertinentes nesta fase de vida. Entidades em saúde, como o American College of Sports Medicine(3) e a Sociedade Brasileira de Medicina do Esporte, em conjunto com a Sociedade Brasileira de Geriatria e Gerontologia(7) sugerem trabalhos de força e potência muscular com volumes e intensidades diferentes, por isso a necessidade de maiores investigações sobre esta temática e uma periodização bem elaborada com aumento progressivo de cargas.

\section{Declaração de conflito de interesses}

Não há nenhum conflito de interesses.

\section{Declaração de financiamento}

Não houve financiamento para o presente estudo.

\section{Referências}

1. IBGE : Instituto Brasileiro de Geografia e Estatística. [Online] Available from: https://ww2.ibge.gov.br/home/estatistica/ populacao/projecao_da_populacao/2013/ default.shtm [Accessed: 26th September 2018]

2. Carvalho J, Soares JMC. Envelhecimento e força muscular: breve revisão. Revista Portuguesa de Ciências do Desporto. 2004;4(3):79-93.

3. American College of Sports Medicine, Chodzko-Zajko WJ, Proctor DN, Fiatarone Singh MA, Minson CT, Nigg $\mathrm{CR}$, et al. American College of Sports Medicine position stand. Exercise and physical activity for older adults. Medicine and Science in Sports and Exercise. [Online] 2009;41(7): 15101530. Available from: doi:10.1249/MSS.0b013e3181a0c95c

4. Diz JBM, Queiroz BZ de, Tavares LB, Pereira LSM. Prevalência de sarcopenia em idosos: resultados de estudos transversais amplos em diferentes países. Revista Brasileira de Geriatria e Gerontologia. [Online] 2015;18(3): 665678. Available from: doi:10.1590/18099823.2015.14139

5. Manini TM, Clark BC. Dynapenia and aging: an update. The Journals of
Gerontology. Series A, Biological Sciences and Medical Sciences. [Online] 2012;67(1): 28-40. Available from: doi:10.1093/gerona/glr010

6. Bottaro M, Machado SN, Nogueira W, Scales R, Veloso J. Effect of high versus low-velocity resistance training on muscular fitness and functional performance in older men. European Journal of Applied Physiology. [Online] 2007;99(3): 257-264. Available from: doi:10.1007/s00421-006-0343-1

7. Nóbrega ACL da, Freitas EV de, Oliveira MAB de, Leitão MB, Lazzoli JK, Nahas $\mathrm{RM}$, et al. Posicionamento oficial da Sociedade Brasileira de Medicina do Esporte e da Sociedade Brasileira de Geriatria e Gerontologia: atividade física e saúde no idoso. Revista Brasileira de Medicina do Esporte. [Online] 1999;5(6): 207-211. Available from: doi:10.1590/S1517-86921999000600002

8. Mariano ER, Navarro F, Sauaia BA, Junior O, De MNS, Marques RF. Força muscular e qualidade de vida em idosas. Revista Brasileira de Geriatria e Gerontologia. [Online] 2013;16(4): 805811. Available from: doi:10.1590/S180998232013000400014

9. Rikli RE, Jones CJ. Development and Validation of a Functional Fitness Test for Community-Residing Older Adults. Journal of Aging and Physical Activity. [Online] 1999;7(2): 129-161. Available from: doi:10.1123/japa.7.2.129

10. Nascimento R, Kanitz A, Kruel L. Efeitos de diferentes estratégias de treinamento combinado na força muscular e na potência aeróbia de idosos: uma revisão sistemática. Revista Brasileira de Atividade Física \& Saúde. [Online] 2015;20(4): 329-329. Available from: doi:10.12820/rbafs.v.20n4p329

11. Tiggemann CL, Dias CP, Noll M, Schoenell MCW, Kruel LFM. Envelhecimento e treinamento de potência: aspectos neuromusculares e funcionais. Revista da Educação Física / UEM. [Online] 2013;24(2): 295-304. 
Available

from:

doi:10.4025/reveducfis.v24.2.15725

12. Cadore EL, Pinto RS, Kruel LFM. Adaptações neuromusculares ao treinamento de força e concorrente em homens idosos. Revista Brasileira de Cineantropometria \& Desempenho Humano. [Online] 2012;14(4): 483-495. Available from: doi:10.5007/19800037.2012v14n4p483

13. Mazini Filho ML, Ferreira RW, César EP. Os benefícios do treinamento físico de força na autonomia funcional do indivíduo idoso. Revista de Educação Física / Journal of Physical Education. [Online] 2006;75(134):57-68. Available from: http://177.38.96.106/index.php/revista/art icle/view/421 [Accessed: 27th September 2018]

14. Skelton DA, Greig CA, Davies JM, Young A. Strength, power and related functional ability of healthy people aged 65-89 years. Age and Ageing. 1994;23(5): 371-377.

15. Mazini Filho ML, Zanella AL, Aidar FJ, Silva AMS da, Salgueiro R da S, Matos DG de. Atividade física e envelhecimento humano: a busca pelo envelhecimento saudável. Revista Brasileira de Ciências do Envelhecimento Humano. [Online] 2010;7(1). Available from: doi:10.5335/rbceh.2012.448 [Accessed: 27th September 2018]

16. Pedro EM, Amorim DB. Análise comparativa da massa e força musculare do equilíbrio entre indivíduos idosos praticantes e não praticantes de musculação. Conexões. [Online] 2008;6(0): 174-183. Available from: doi:10.20396/conex.v6i0.8637823

17. Aagaard P. Training-induced changes in neural function. Exercise and Sport Sciences Reviews. 2003;31(2): 61-67.

18. Fleck SJ, Kraemer WJ. Fundamentos do treinamento de força muscular. 4. ed. Porto Alegre: Artmed; 2017.

19. Tiggemann CL, Dias CP, Radaelli R, Massa JC, Bortoluzzi R, Schoenell MCW, et al. Effect of traditional resistance and power training using rated perceived exertion for enhancement of muscle strength, power, and functional performance. Age (Dordrecht, Netherlands). [Online] 2016;38(2): 42. Available from: doi:10.1007/s11357-0169904-3

20. Ueno DT, Gobbi S, Teixeira CVL, Sebastião É, Prado AKG, Costa JLR, et al. Effects of three methods of physical activity in functional ability of elderly. Revista Brasileira de Educação Física e Esporte. [Online] 2012;26(2): 273-281. Available from: doi:10.1590/S180755092012000200010

21. Moraes K, Correa CS, Pinto RS, Schuch F, Radaelli R, Gaya A, et al. Efeitos de três programas de treinamento de força na qualidade de vida de idosas. Revista Brasileira de Atividade Física \& Saúde. [Online] 2012;17(3): 181-187. Available from: doi:10.12820/rbafs.v.17n3p181-187

22. Lustosa LP, Silva JP, Coelho FM, Pereira DS, Parentoni AN, Pereira LSM. Efeito de um programa de resistência muscular na capacidade funcional e na força muscular dos extensores do joelho em idosas préfrágeis da comunidade: ensaio clínico aleatorizado do tipo crossover. Brazilian Journal of Physical Therapy. [Online] 2011;15(4): 318-324. Available from: doi:10.1590/S1413-35552011000400010

23. Mezzaroba PV, Prati ARC. Influence of strength training on variables related to elderly autonomy - doi 10.4025/actascihealthsci.v34i2.8927.

Acta Scientiarum. Health Sciences. [Online] 2012;34(2): 157-162. Available from:

doi:10.4025/actascihealthsci.v34i2.8927

24. Prado RA, Teixeira ALC, Langa CJS, Egydio PRM, Izzo P. A influência dos exercícios resistidos no equilíbrio, mobilidade funcional e na qualidade de vida de idosas. Mundo Saúde. 2010;34(2):183-91.

25. Cabral ACA, Moreira Magalhães ÍK, Borba-Pinheir CJ, de Macêdo Barreto da Rocha-Júnior OR, Almeida de Figueiredo NM, Matin Dantas H. Composição corporal e autonomia funcional de 
mulheres idosas após um programa de treinamento resistido. Revista de Pesquisa Cuidado Fundamental. [Online] 2014;6(1). Available from: http://www.redalyc.org/resumen.oa?id=5 05750621007 [Accessed: 27th September 2018].

26. Andrade RM, Matsudo SMM. Relação da força explosiva e potência muscular com a capacidade funcional no processo de envelhecimento. Revista Brasileira de Medicina do Esporte. [Online] 2010;16(5): 344-348. Available from: doi:10.1590/S1517-86922010000500005

27. Ceccato M, Gurjão A, Prado A, Gallo L, Filho JJ, Gobbi S. Treinamento com pesos, velocidade de movimento e desempenho muscular: uma revisão sistemática. Revista Brasileira de Atividade Física \& Saúde. [Online] 2013;18(5): 536-536. Available from: doi:10.12820/rbafs.v.18n5p536

28. Correa CS, Pinto RS. Efeitos de diferentes tipos de treinamento de força no desempenho de capacidades funcionais em mulheres idosas. Estudos Interdisciplinares sobre o Envelhecimento. [Online] 2010;16(1). Available from: http://seer.ufrgs.br/RevEnvelhecer/article/ view/13300 [Accessed: 27th September 2018].

29. Dantas EHM, Figueira HA, Emygdio RF, Vale RGS. Functional autonomy GDLAM protocol classification pattern in elderly women. Indian Journal Applied Research. 2014;4(7):262-6.

30. Dantas EHM, Vale, RGS. Protocolo GDLAM de avaliação da autonomia funcional. Fitness \& Performance Journal. 2004;3(3):175-82.

31. Tschopp M, Sattelmayer MK, Hilfiker R. Is power training or conventional resistance training better for function in elderly persons? A meta-analysis. Age and Ageing. [Online] 2011;40(5): 549-556. Available from: doi:10.1093/ageing/afr005
32. Csapo R, Alegre LM. Effects of resistance training with moderate vs heavy loads on muscle mass and strength in the elderly: A meta-analysis. Scandinavian Journal of Medicine \& Science in Sports. [Online] 2016;26(9): 995-1006. Available from: doi:10.1111/sms.12536 